\title{
3D Eulerian simulation of a gas-solid bubbling fluidized bed: assessment of drag coefficient correlations
}

\author{
E. Esmaili \& N. Mahinpey \\ Department of Chemical and Petroleum Engineering, \\ Schulich School of Engineering, University of Calgary, Canada
}

\begin{abstract}
Fluidized beds have been widely used in power generation and in the chemical, biochemical, and petroleum industries. The 3D simulation of commercial scale fluidized beds has been computationally impractical due to the required memory and processor speeds. However, in this study, 3D Computational Fluid Dynamics simulation of a gas-solid bubbling fluidized bed is performed to investigate the effect of using different inter-phase drag models. The drag correlations of Syamlal-O'Brien, Gidaspow, and Wen-Yu are reviewed using a multiphase Eulerian-Eulerian model to simulate the momentum transfer between phases. Comparisons are made with both a 2D Cartesian simulation and experimental data. The experiments are performed on a Plexiglas rectangular fluidized bed consisting of spherical glass beads and ambient air as the gas phase. The aim of this work is to present an optimum drag model to simulate the momentum transfer between phases and compare the results using $3 \mathrm{D}$ versus $2 \mathrm{D}$ simulation of gas-solid bubbling fluidized beds. Comparisons were made based on solid volume fractions, expansion height, and pressure drop inside the fluidized bed at different superficial gas velocities. The results were found to agree well with experimental data.

Keywords: multiphase flow, fluidized bed, computational fluid dynamics, interphase drag model.
\end{abstract}

\section{Introduction}

Two approaches are typically used for Computational Fluid Dynamics (CFD) modeling of gas-solid fluidized beds. The first one is Lagrangian-Eulerian 
modeling [1,2,7,10-12], which solves the equations of motion for each particle individually and uses a continuous interpenetrating model (Eulerian framework) for modeling the gas phase. As a consequence, the Lagrangian-Eulerian model requires large computational resources for large systems of particles. The second method is Eulerian-Eulerian modeling [4,9,12-16], which assumes both phases can be considered as fluid and also considers the interpenetrating effect of each phase by using drag models. Therefore, applying a proper drag model in Eulerian-Eulerian modeling is of a great importance.

Many researchers have applied 2D Cartesian simulations to model pseudo-2D beds $[1,4,6,15]$. Peirano et al. [5] have investigated the importance of three dimensionality in the Eulerian approach simulations of stationary bubbling fluidized beds. The results of their simulations show that two-dimensional simulations should be used with caution and only for sensitivity analysis, whereas three-dimensional simulations are able to reproduce both the statics (bed height and spatial distribution of particles) and the dynamics (power spectrum of pressure fluctuations) of the bed. In addition, they believe that the issue of accurate prediction of the drag force (the force exerted by the gas on a single particle in a suspension) does not seem to be the most important problem when dealing with bubbling beds where accurate empirical correlations are available. In contrast, in the present work, it is found that using a proper drag model can increase the accuracy of results in $3 \mathrm{D}$ simulation of bubbling fluidized beds. Goldschmidt et al. [6] applied a two-dimensional multi-fluid Eulerian CFD model to study the influence of the coefficient of restitution on the hydrodynamics of dense gas-solid fluidized beds. They showed that, in order to obtain realistic bed dynamics from fundamental hydrodynamic models, it is of prime importance to correctly take the effect of energy dissipation due to nonideal particle-particle encounters into account. van Wachem et al. [8] implemented a CFD model for a free bubbling fluidized bed in the commercial code CFX of AEA Technology to verify experimentally Eulerian-Eulerian gassolid model simulations of bubbling fluidized beds with existing correlations for bubble size and bubble velocity. They concluded that smaller bubbles and a lower rise velocity are usually observed with 2D beds. Cammarata et al. [9] compared the bubbling behavior predicted by 2D and 3D simulations of a rectangular fluidized bed using commercial software, CFX. The bed expansion, bubble hold-up, and bubble size calculated from the 2D and 3D simulations were compared with the predictions obtained from the Darton equation [3]. A more realistic physical behavior model of fluidization was obtained using 3D simulations. They also indicate that $2 \mathrm{D}$ simulations could be used to conduct sensitivity analyses.

Xie et al. [14] compared the results of 2D and 3D simulation of slugging, bubbling, and turbulent gas-solid fluidized beds. They also investigated the effect of using different coordinate systems. Their results show that there is a significant difference between 2D and 3D simulations, and only 3D simulations could predict the correct bed height and pressure spectra. Behjat et al. [15] applied a two dimensional CFD technique in order to investigate hydrodynamic and heat transfer phenomena. They conclude that a Eulerian-Eulerian model is 
suitable for modeling industrial fluidized bed reactors. Their results indicate that considering two solid phases, particles with smaller diameters have lower volume fraction at the bottom of the bed and higher volume fraction at the top of the bed and also that the gas temperature increases as it moves upward in the reactor due to the heat of polymerization reaction leading to the higher temperatures at the top of the bed. Li et al. [16] conducted a three-dimensional numerical simulation of a single horizontal gas jet into a laboratory-scale cylindrical gas-solid fluidized bed. They proposed a scaled drag model and implemented it into the simulation of a fluidized bed of FCC particles. They also obtained the jet penetration lengths of different jet velocities and compared them with published experimental data, as well as with predictions of empirical correlations.

No previous works in the literature have investigated the effect of using different drag models in 3D simulation of fluidized beds to present an optimum drag model for simulation of bubbling gas-solid fluidized beds. In this respect, the underlying objective of this study is to present an optimum drag model to simulate the momentum transfer between phases and compare the results using $3 \mathrm{D}$ versus $2 \mathrm{D}$ simulation of gas-solid bubbling fluidized beds.
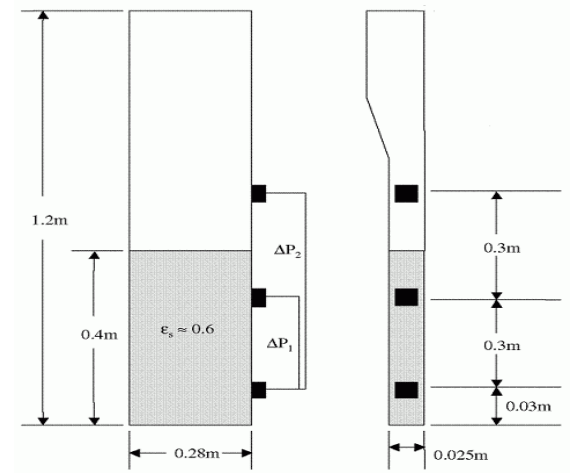

Figure 1: Geometry of 3D Plexiglas fluidized bed.

\section{Experimental setup}

Experiments were carried out in the Department of Chemical and Biological Engineering at the University of British Columbia. The Column is a psudo-2D Plexiglas of $1.2 \mathrm{~m}$ height, $0.28 \mathrm{~m}$ width, and $0.025 \mathrm{~m}$ thickness. Spherical glass beads of 250-300 $\mu \mathrm{m}$ diameter and density $2500 \mathrm{~kg} / \mathrm{m}^{3}$ were fluidized with air at ambient conditions. Pressure drops were measured using three differential pressure transducers located at elevation $0.03,0.3$, and $0.6 \mathrm{~m}$ above the gas distributor, respectively. The static bed height of $0.4 \mathrm{~m}$ with a solid volume fraction of 0.6 was used in all the experiments. Pressure drop and bed expansion were monitored at different superficial gas velocities ranging from 0 to $0.8(\mathrm{~m} / \mathrm{s})$. 


\section{Governing equations}

The proposed model's equations, which are solved numerically, are presented in Table 1 .

\section{Numerical simulation}

Governing equations of mass and momentum conservation are solved using finite volume method employing the Semi Implicit Method for Pressure Linked Equations (PC-SIMPLE) algorithm, which is an extension of the SIMPLE algorithm to multiphase flow. A multi fluid Eulerian-Eulerian model, which considers the conservation of mass and momentum for the gas and solid phases, was applied. The kinetic theory of granular flow, which considers the conservation of solid fluctuation energy, was used for closure of the solids stress terms. The three-dimensional (3D) geometry has been meshed using 336,000 structured rectangular cells. Volume fraction, density, and pressure are stored at the main grid points that are placed in the center of each control volume. A staggered grid arrangement is used, and the velocity components are solved at the control volume surfaces. A pressure correction equation is built based on total volume continuity. Pressure and velocities are then corrected so as to satisfy the continuity constraint. A grid sensitivity analysis is performed using different mesh sizes and $2 \mathrm{~mm}$ mesh interval spacing was chosen for all the simulation runs. Second-order upwind discretization schemes were used for discretizing the governing equations. Based on the estimation of the truncation error, an adaptive time-stepping algorithm with 100 iterations per each time step and a minimum value of order $10^{-5}$ for the lower domain of time step was used to ensure a stable convergence. The convergence criteria for other residual components associated with the relative error between two successive iterations has been specified in the order of $10^{-5}$. Three different drag models are studied in this work to simulate the momentum transfer between phases (Gidaspow, Syamlal-O'Brien, and Wen-Yu.). FLUENT 6.3 on a 20 AMD/Opteron 64bit processor Sun Grid Microsystems workstation W2100Z with 4 GB RAM is employed to solve the governing equations. Computational model parameters are listed in Table.2.

\section{Results and discussion}

Simulation results were compared with the experimental data in order to validate the model. Figure 2 shows the time average pressure drop inside the bed between two specific elevations (i.e. $0.03 \mathrm{~m}$ and $0.3 \mathrm{~m}$ as demonstrated in Fig. 1) for different studied cases and experimental results. In order to calculate the pressure at each pressure sensor (i.e. $y=0.03 \mathrm{~m}$ ), two kinds of averaging have been applied. The first one is the spatial averaging, which is the average value of pressure for all nodes in the plane of first pressure sensor (plane $y=0.03$ ). The second one is the time averaging of spatial-averaged pressure values in the period of 3-10 sec real time. As indicated in Fig. 2, the pressure drop for all the 
Table 1: $\quad$ Governing equations.

Continuity:

Momentum equations:

$$
\begin{gathered}
\frac{\partial}{\partial t}\left(\varepsilon_{g} \rho_{g}\right)+\nabla \cdot\left(\varepsilon_{g} \rho_{g} \vec{V}_{g}\right)=0 \\
\frac{\partial}{\partial t}\left(\varepsilon_{s} \rho_{s}\right)+\nabla \cdot\left(\varepsilon_{s} \rho_{s} \vec{V}_{s}\right)=0
\end{gathered}
$$

$$
\begin{gathered}
\frac{\partial}{\partial t}\left(\varepsilon_{g} \rho_{g} \vec{V}_{g}\right)+\nabla \cdot\left(\varepsilon_{g} \rho_{g} \vec{V}_{g} \vec{V}_{g}\right)=\nabla \cdot \overline{\bar{\tau}}_{g}-\varepsilon_{g} \nabla P+\varepsilon_{g} \rho_{g} g+K_{s g}\left(\vec{V}_{s}-\vec{V}_{g}\right) \\
\frac{\partial}{\partial t}\left(\varepsilon_{s} \rho_{s} \vec{V}_{s}\right)+\nabla \cdot\left(\varepsilon_{s} \rho_{s} \vec{V}_{s} \vec{V}_{s}\right)=\nabla \cdot \overline{\bar{\tau}}_{s}-\nabla P_{s}-\varepsilon_{s} \nabla P+\varepsilon_{s} \rho_{s} g+K_{s g}\left(\vec{V}_{s}-\vec{V}_{g}\right)
\end{gathered}
$$

Solid pressure

$$
P_{S}=\varepsilon_{s} \rho_{s} \Theta_{s}\left[1+2 g_{0} \varepsilon_{s}(1+e)\right]
$$

Radial distribution function

$$
g_{0}=\frac{3}{5}\left[1-\left(\frac{\varepsilon_{S}}{\varepsilon_{s, \max }}\right)^{\frac{1}{3}}\right]^{-1}
$$

\section{Granular temperature}

Stress tensors

$$
\begin{gathered}
\Theta_{S}=\left[\frac{-\left(K_{1} \varepsilon_{S}+\rho_{s}\right) \operatorname{Tr}\left(\overline{\bar{D}}_{S}\right)}{2 K_{4} \varepsilon_{S}}+\frac{\sqrt{\left(K_{1} \varepsilon_{S}+\rho_{s}\right)^{2} \operatorname{Tr}^{2}\left(\overline{\bar{D}}_{S}\right)+4 K_{4} \varepsilon_{S}\left[2 K_{3} \operatorname{Tr}\left(\overline{\bar{D}}_{s}^{2}\right)+K_{2} \operatorname{Tr}^{2}\left(\overline{\bar{D}}_{S}\right)\right]}}{2 K_{4} \varepsilon_{s}}\right]^{2} \\
K_{1}=2(1-e) \rho_{s} g_{0} \\
K_{3}=\frac{d_{s} \rho_{s}}{2}\left\{\frac{K_{2}=\frac{4}{3 \sqrt{\pi}} d_{s} \rho_{s}(1+e) g_{0} \varepsilon_{s}-\frac{2}{3} K_{3}}{3(3-e)}\left[1+\frac{2}{5}(1+e)(3 e-1) g_{0} \varepsilon_{s}\right]+\frac{8 \varepsilon_{s}}{5 \sqrt{\pi}} g_{0}(1+e)\right\} \\
K_{4}=\frac{12\left(1-e^{2}\right) \rho_{s} g_{0}}{d_{s} \sqrt{\pi}}
\end{gathered}
$$

$$
\begin{gathered}
\overline{\bar{\tau}}_{g, e f f}=\varepsilon_{g}\left(\overline{\bar{\tau}}_{g}+\overline{\bar{\tau}}_{t}\right) \\
\overline{\bar{\tau}}_{g}=\mu_{g}\left(\nabla \vec{V}_{g}+\left(\nabla \vec{V}_{g}\right)^{T}\right)-\frac{2}{3} \mu_{g} \overline{\bar{I}} \cdot \nabla \cdot \vec{V}_{g} \\
\overline{\bar{\tau}}_{t}=\mu_{t}\left(\nabla \vec{V}_{g}+\left(\nabla \vec{V}_{g}\right)^{T}\right)-\frac{2}{3} \mu_{t} \overline{\bar{I}} \cdot \nabla \cdot \vec{V}_{g}-\frac{2}{3} \varepsilon_{g} \rho_{g} k \overline{\bar{I}} \\
\overline{\bar{\tau}}_{s}=\mu_{s}\left(\nabla \vec{V}_{g}+\left(\nabla \vec{V}_{g}\right)^{T}\right)-\left(\lambda_{s}-\frac{2}{3} \mu_{s}\right) \overline{\bar{I}} \cdot \nabla \cdot \vec{V}_{g} \\
\mu_{s}=\frac{4}{5} \varepsilon_{s}{ }^{2} \rho_{s} d_{s} g_{0}(1+e) \sqrt{\frac{\Theta}{\pi}}+\frac{\frac{5 \sqrt{\pi}}{48} \rho_{s} d_{s} \sqrt{\Theta}}{(1+e) g_{0}}\left[1+\frac{4}{5} g_{0} \varepsilon_{s}(1+e)\right]^{2}
\end{gathered}
$$


Table 1: $\quad$ Continued.

Inter-phase momentum exchange

$$
\lambda_{s}=\frac{4}{3} \varepsilon_{s}^{2} \rho_{s} d_{s} g_{0}(1+e) \sqrt{\frac{\Theta}{\pi}}
$$

Gidaspow [17]

$$
\begin{gathered}
K_{s g}=\left\{\begin{array}{cc}
150 \frac{\varepsilon_{s}{ }^{2} \mu_{g}}{\varepsilon_{g} d_{s}{ }^{2}+1.75 \frac{\varepsilon_{s} \rho_{g}\left|\vec{V}_{s}-\vec{V}_{g}\right|}{d_{s}}} \quad \text { if } \quad \varepsilon_{s}>0.2 \\
\frac{3}{4} C_{d} \varepsilon_{g}-2.65 \frac{\varepsilon_{s} \varepsilon_{g} \rho_{g}\left|\vec{V}_{s}-\vec{V}_{g}\right|}{d_{s}} \quad \text { if } \quad \varepsilon_{s} \leq 0.2
\end{array}\right. \\
C_{d}=\left\{\begin{array}{lll}
\frac{24}{R e . \varepsilon_{g}}\left(1+0.15\left(\text { Re. } \varepsilon_{g}\right)^{0.687}\right) & \text { if } & \text { Re. } \varepsilon_{g}<1000 \\
0.44 & \text { if } \quad \text { Re. } \varepsilon_{g} \geq 1000
\end{array}\right. \\
R e=\frac{\rho_{g\left|\vec{V}_{s}-\vec{V}_{g}\right| d_{s}}}{\mu_{g}}
\end{gathered}
$$

Syamlal and O'Brien [18]$$
\begin{gathered}
K_{s g}=\frac{3}{4} C_{d} \frac{\varepsilon_{s} \varepsilon_{g} \rho_{g}}{V_{r}^{2} d_{s}}\left|\vec{V}_{s}-\vec{V}_{g}\right| \\
C_{d}=\left(0.63+4.8 \sqrt{\frac{V_{r}}{R e}}\right)^{2} \\
V_{r}=0.5\left[a-0.06 R e+\sqrt{(0.06 R e)^{2}+0.12 \operatorname{Re}(2 b-a)+a^{2}}\right] \\
a=\varepsilon_{g}^{4.14} \\
b=\left\{\begin{array}{cll}
P \varepsilon_{g}{ }^{1.28} & \text { if } & \varepsilon_{g} \leq 0.85 \\
\varepsilon_{g} Q^{Q} & \text { if } & \varepsilon_{g} \leq 0.85
\end{array}\right.
\end{gathered}
$$$$
P=0.8 \text { and } Q=2.65 \text { by default }
$$

Wen $Y u[19]$

$$
\begin{aligned}
& K_{s g}=\frac{3}{4} C_{d}{ }^{-2.65} \frac{\varepsilon_{s} \varepsilon_{g} \rho_{g}}{d_{s}}\left|\vec{V}_{s}-\vec{V}_{g}\right| \\
& C_{d}= \begin{cases}\frac{24}{R e . \varepsilon_{g}}\left(1+0.15\left(\text { Re. } \varepsilon_{g}\right)^{0.687}\right) & \text { if } \quad \text { Re. } \varepsilon_{g}<1000 \\
0.44 & \text { if } \quad \text { Re. } \varepsilon_{g} \geq 1000\end{cases} \\
& R e=\frac{\rho_{g\left|\vec{V}_{s}-\vec{V}_{g}\right| d_{s}}}{\mu_{g}}
\end{aligned}
$$


Table 2: $\quad$ Computational model parameters.

\begin{tabular}{|c|c|c|}
\hline Parameter & & Value \\
\hline Particle density & & $2500\left(\mathrm{~kg} / \mathrm{m}^{3}\right)$ \\
\hline Gas density & & $1.225\left(\mathrm{~kg} / \mathrm{m}^{3}\right)$ \\
\hline Mean particle diameter & & $275 \mu m$ \\
\hline Initial solid packing & & 0.6 \\
\hline Superficial gas velocity & & $11.7,21,38,46(\mathrm{~cm} / \mathrm{s})$ \\
\hline Bed dimension & $0.28(m)$ & $\times 1.2(\mathrm{~m}) \times 0.025(\mathrm{~m})$ \\
\hline Static bed height & & $0.4(m)$ \\
\hline Grid interval spacing & & $0.002(m)$ \\
\hline Inlet boundary condition type & & Inlet-velocity \\
\hline $\begin{array}{l}\text { Outlet boundary condition } \\
\text { type }\end{array}$ & & Pressure-outlet \\
\hline \multirow{4}{*}{ Under-relaxation factors } & Pressure & 0.6 \\
\hline & Momentum & 0.4 \\
\hline & Volume fraction & 0.3 \\
\hline & Granular temperature & 0.2 \\
\hline
\end{tabular}

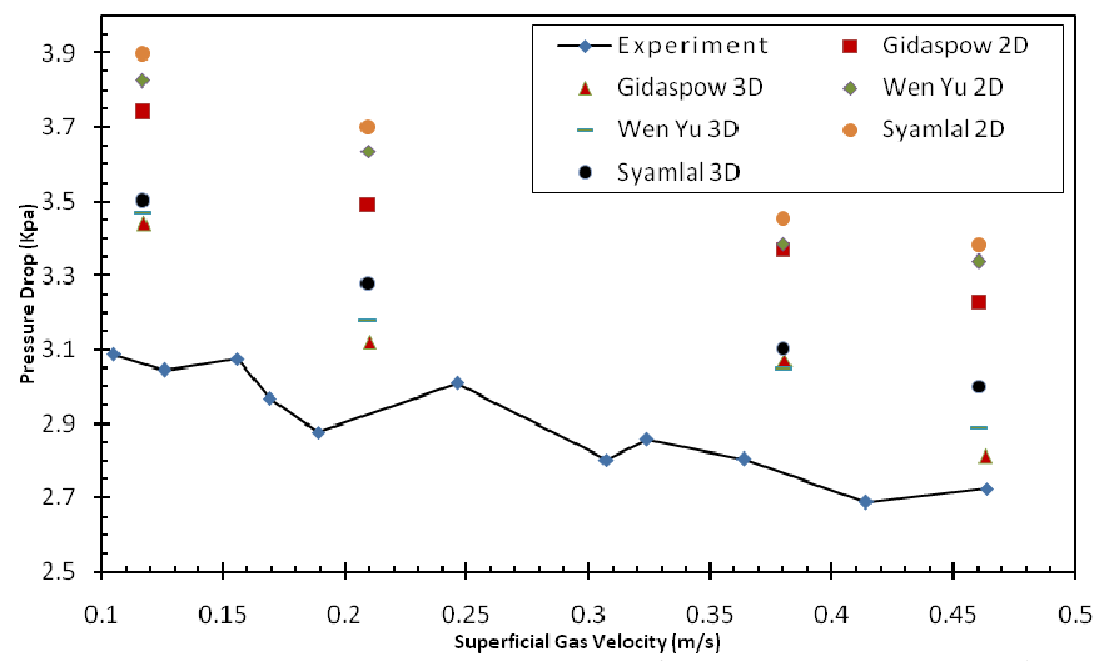

Figure 2: $\quad$ Pressure drop inside the bed $\left(\Delta P_{1}=\bar{P}_{Z=0.03 m}-\bar{P}_{Z=0.3 m}\right)$.

models showed a declining trend with increase of the superficial gas velocity, which is in good qualitative agreement with the experimental data. It can be easily seen that $3 \mathrm{D}$ simulations show their superiority in predicting the pressure drop inside the bed compared with 2D simulation. Also, it can be concluded that the Gidaspow drag model in a 3D simulation will give results closer to the experimental data. 
The experimental data of the time-average bed expansion ratio were compared with corresponding values predicted by the model, using SyamlalO'Brien, Gidaspow, and Wen-Yu drag functions for various velocities as depicted in Figure 3. All drag models demonstrate a consistent increase in bed expansion with gas velocity and predict the bed expansion reasonably well. Figure 3 shows the considerable relative increase in bed expansion as the fluidizing velocity increases; a $5 \%$ increase was obtained at $0.11 \mathrm{~m} / \mathrm{s}$, a $20 \%$ increase at $0.21 \mathrm{~m} / \mathrm{s}$, and $42 \%$ at $0.38 \mathrm{~m} / \mathrm{s}$, and up to a $50 \%$ increase in bed height was measured at $0.46 \mathrm{~m} / \mathrm{s}$, the highest fluidized velocity investigated. It can be seen that using a $3 \mathrm{D}$ simulation, especially for lower superficial gas velocities, will increase the accuracy of the results. The reason can be the effect of participating governing equations in the $\mathrm{z}$ direction (depth of the bed) in simulating the fluid flow when the gas velocity increases. It is also seen that using the Gidaspow drag model in 3D simulation of a gas-solid fluidized bed will give better results for predicting the bed expansion ratio than the other two drag models.

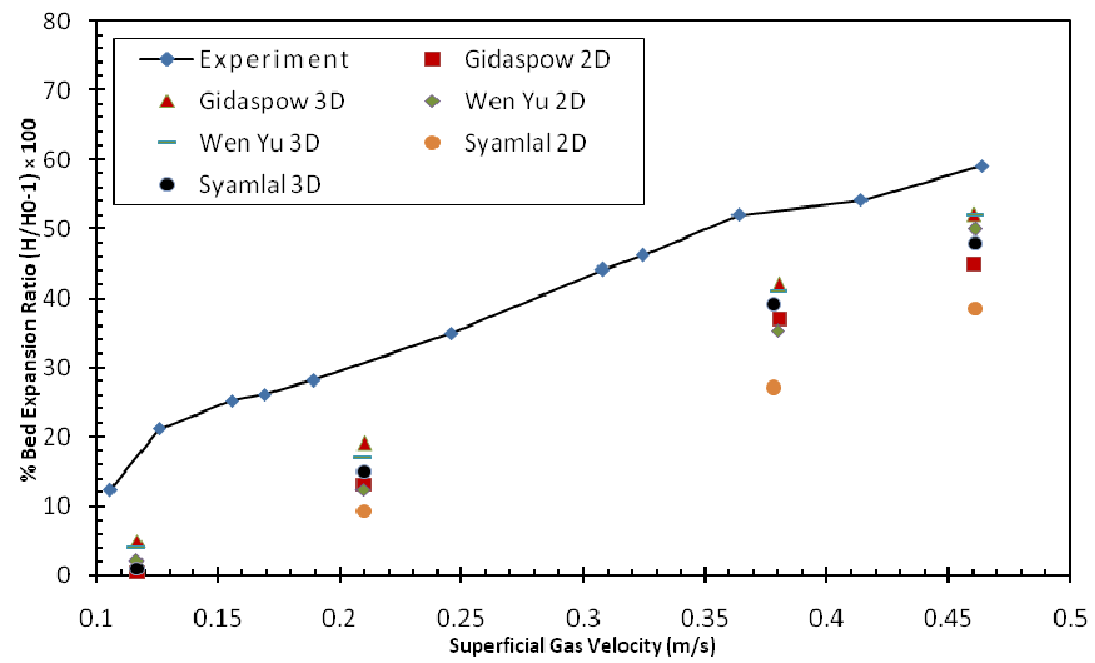

Figure 3: Comparison of simulated bed expansion ratio with experimental data.

Figure 4 shows a contour plot of solid volume fraction for the three drag models studied in this work for a superficial gas velocity of $0.38 \mathrm{~m} / \mathrm{sec}$ at $10 \mathrm{sec}$ real-time simulations. As can be observed from the plots, the Syamlal model represents the lowest bed expansion and gas void fraction. This fact could have been foreseen from the minimum fluidization velocity prediction of this model, which is almost five times larger than experimental data reported in the literature. The rest of the models showed approximately the same range of bed expansion. Expansion of the bed started with the formation of bubbles for all the 


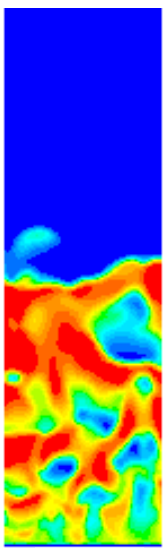

(a)

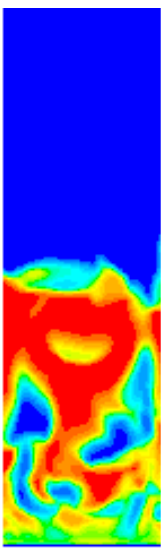

(b)

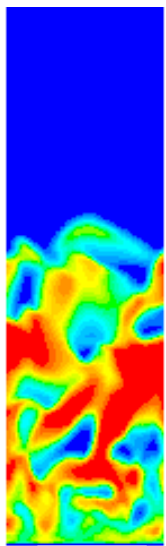

(c)

Figure 4: Contours of solid volume fraction at $\mathrm{t}=10 \mathrm{~s}$ and $\mathrm{u}=0.38 \mathrm{~m} / \mathrm{s}$ (a) Syamlal (b) Wen Yu (c) Gidaspow.

models and eventually reached a statistically steady-state bed height. After this point, an unsteady chaotic generation of bubbles was observed after almost 3 seconds of real time simulation.

\section{Conclusion}

Numerical simulation of a bubbling gas-solid fluidized bed have been performed in a three dimensional solution domain using the Eulerian-Eulerian approach to investigate the effect of using three dimensional analysis versus two dimensional simulation of fluidized beds. FLUENT 6.3 was used to perform the calculations. The results show that although three-dimensional simulation takes more time and computing processors than two-dimensional simulation, it gives more accurate results when the models are compared with experimental data. Also, a comparison between three common drag models, Syamlal, Wen Yu, Gidaspow, was performed to develop an optimized drag model for simulation of momentum transfer between phases in a 3D bubbling gas-solid fluidized bed. It is concluded that the Gidaspow drag model achieved better results in predicting the bed expansion ratio and pressure drop inside the bed than Syamlal drag correlations. However, further modeling efforts are required to study the influence of using other drag models, which have not been studied, and optimizing existing drag models based on minimum fluidization velocity in three dimensional simulations will be performed in future work.

\section{Nomenclature}

$C_{d} \quad$ Single particle drag function, dimensionless

$\overline{\bar{D}}_{s} \quad$ Rate of strain tensor 
$d_{s} \quad$ Solid diameter, $\mathrm{m}$

$e \quad$ Restitution coefficient, dimensionless

$g$ Acceleration due to gravity, $\mathrm{m} / \mathrm{s}^{2}$

$g_{0} \quad$ Radial distribution coefficient, dimensionless

$K_{s g} \quad$ Gas/solid momentum exchange coefficient, dimensionless

$P \quad$ Pressure, $\mathrm{Pa}$

$P_{S} \quad$ Solid pressure, $\mathrm{Pa}$

$\vec{V} \quad$ Velocity, $\mathrm{m} / \mathrm{s}$

Re Reynolds number, dimensionless

Greek symbols

$\rho \quad$ Density, $\mathrm{kg} / \mathrm{m}^{3}$

$\Theta_{s} \quad$ Granular temperature, $\mathrm{m}^{2} / \mathrm{s}^{2}$

$\overline{\bar{\tau}} \quad$ Stress tensor, $\mathrm{Pa}$

$\mu \quad$ Shear viscosity, $\mathrm{kg} / \mathrm{sm}$

$\lambda$ Bulk viscosity, $\mathrm{kg} / \mathrm{sm}$

$\varepsilon \quad$ Volume fraction, dimensionless

\section{Subscripts}

$\begin{array}{ll}g & \text { Gas } \\ s & \text { Solid }\end{array}$

\section{References}

[1] B.P.B. Hoomans, J.A.M. Kuipers, W.J. Briels, V.W.P.M. Swaaij, Discrete particle simulation of bubble and slug formulation in a two-dimensional gas-fluidized bed: a hard-sphere approach, Chem. Eng. Sci. 51 (1996) 99.

[2] B. Xu, A. Yu, Numerical simulation of the gas-solid flow in a fluidized bed by combining discrete particle method with computational fluid dynamics, Chem. Eng. Sci. 52 (1997) 2785.

[3] R.C. Darton, R.D. LaNauZe, J.F. Davidson, D. Harrison, Bubble growth due to coalescence in fluidized beds, Trans. Inst. Chem. Eng. 55 (1977) 274.

[4] B.G.M. van Wachem, J.C. Schouterf, R. Krishnab, and C.M. van den Bleek, Eulerian Simulations of Bubbling Behavior in Gas-Solid Fluidized Beds, Computers chem. Engng Vol. 22, Suppl., pp. S 299-S306. 1998.

[5] E. Peiranoa, V. Delloumea, B. Lecknera, Two- or three-dimensional simulations of turbulent gas-solid flows applied to fluidization, Chemical Engineering Science 56 (2001) 4787-4799.

[6] M. J. V. Goldschmidt, J. A. M. Kuipers, W. P. M. van Swaaij, Hydrodynamic modeling of dense gas-fluidized beds using the kinetic theory of granular flow: effect of coefficient of restitution on bed dynamics, Chemical Engineering Science 56 (2001) 571-578. 
[7] K.D. Kafui, C. Thornton, M.J. Adams, Discrete particle-continuum fluid modeling of gas-solid fluidized beds, Chem. Eng. Sci. 57 (2002) 2395.

[8] W.B.G.W. Wachem, A.E. Almstedt, Methods for multiphase computational fluid dynamics, Chem. Eng. J. 96 (2003) 81.

[9] L. Cammarata, P. Lettieri, G.D.M. Micale, D. Colman, 2d and 3d cfd simulations of bubbling fluidized beds using Eulerian-Eulerian models, Int. J. Chem. Reactor Eng. 1 (2003) (Article A48).

[10] M.J.V. Goldschmidt, R. Beetstra, J.A.M. Kuipers, Hydrodynamic modeling of dense gas-fluidized beds: comparison and validation of $3 \mathrm{~d}$ discrete particle and continuum models, Powder Technol. 142 (2004) 23.

[11] J.S. Curtis, V.B. Wachem, Modeling particle-laden flows: a research outlook, AIChE J. 50 (2004) 2638.

[12] M. Chiesa, V. Mathiesen, J. A. Melheim, B. Halvorsen, Numerical simulation of particulate flow by the Eulerian-Lagrangian and the Eulerian-Eulerian approach with application to a fluidized bed. Comput. Chem. Eng. 29 (2005) 291.

[13] J. Sun, F. Battaglia, Hydrodynamic modeling of particle rotation for segregation in bubbling gas-fluidized beds, Chem. Eng. Sci. 61 (2006) 1470.

[14] N. Xie, F. Battaglia, S. Pannala, Effects of using two-versus threedimensional computational modeling of fluidized beds Part I, hydrodynamics, Powder Technology 182 (2008).

[15] Y. Behjat, S. Shahhosseini, S. H. Hashemabadi, CFD modeling of hydrodynamic and heat transfer in fluidized bed reactors, International Communications in Heat and Mass Transfer 35 (2008) 357-368.

[16] T. Li, K. Pougatch, M. Salcudean, D. Grecov, Numerical simulation of horizontal jet penetration in a three-dimensional fluidized bed, Powder Technology 184 (2008) 89-99.

[17] D. Gidaspow, Multiphase Flow and Fluidization: Continuum and Kinetic Theory Descriptions, Academic Press, Boston, 1994.

[18] M. Syamlal, T.J. O'Brien, Computer simulation of bubbles in a fluidized bed, AIChE Symposium Series 85 (1989) 22-31.

[19] C.Y. Wen, Y.H. Yu, Mechanics of fluidization, Chemical Engineering Progress Symposium Series, 1966, pp. 100-111. 